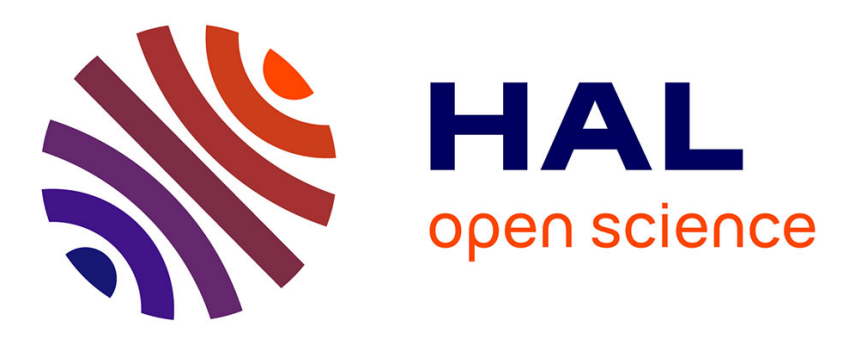

\title{
On the equivalence of multiparameter Gaussian processes
}

Tommi Sottinen, Ciprian A. Tudor

\section{To cite this version:}

Tommi Sottinen, Ciprian A. Tudor. On the equivalence of multiparameter Gaussian processes. Journal of Theoretical Probability, 2006, 19 (2), pp.461-485. 10.1007/s10959-006-0022-5 . hal-00130640

\section{HAL Id: hal-00130640 https://hal.science/hal-00130640}

Submitted on 13 Feb 2007

HAL is a multi-disciplinary open access archive for the deposit and dissemination of scientific research documents, whether they are published or not. The documents may come from teaching and research institutions in France or abroad, or from public or private research centers.
L'archive ouverte pluridisciplinaire HAL, est destinée au dépôt et à la diffusion de documents scientifiques de niveau recherche, publiés ou non, émanant des établissements d'enseignement et de recherche français ou étrangers, des laboratoires publics ou privés. 


\title{
On the equivalence of multiparameter Gaussian processes
}

\author{
Tommi Sottinen \\ Department of Mathematics and Statistics \\ University of Helsinki \\ P.O. Box 68, 00014 Helsinki \\ Finland
}

\author{
Ciprian A. Tudor ${ }^{1}$ \\ Laboratoire de Probabilités \\ Université de Paris 6 \\ 4, place Jussieu, 75252 Paris \\ France
}

\begin{abstract}
Our purpose is to characterize the multiparameter Gaussian processes, that is Gaussian sheets, that are equivalent in law to the Brownian sheet and to the fractional Brownian sheet. We survey multiparameter analogues of the Hitsuda, Girsanov and Shepp representations. As an application, we study a special type of stochastic equation with linear noise.
\end{abstract}

Mathematics Subject Classification (2000): Primary 60G15; Secondary 60G30, 60H05

Key words and phrases: Brownian sheet, Fractional Brownian sheet, equivalence of Gaussian processes, Hitsuda representation, Shepp representation, canonical representation of Gaussian processes, Girsanov theorem, stochastic differential equations

\section{Introduction}

The question of the equivalence in law of Gaussian processes has been widely studied in the sixties-seventies (see [6], [5], [9], [14]). Recently, the problem has been reopened by several authors, due to the intensive study of the fractional Brownian and of stochastic calculus with respect to this process. Precisely, the Wiener integral representation of the fractional Brownian motion with respect to the Brownian motion and the explicit form of its kernel, allows us to find concrete expressions of Gaussian processes that are equivalent in law to the fractional Brownian motion. We refer to [1], [15] and [2] for recent works on the Gaussian equivalence. We investigate in this paper the equivalence in law of multiparameter Gaussian processes (Note that

\footnotetext{
${ }^{1}$ From September 2004 the authors's address will be SAMOS-MATISSE, Université de Paris 1, 90 rue de Tolbiac, 75634 Paris Cedex 13, France.
} 
lately the stochastic integration and stochastic equations with fractional Brownian sheet have been considered in e.g. [4] and [16]). More precisely, we will characterize all processes that are equivalent in law with a Brownian sheet and to a fractional Brownian sheet. We study both Hitsuda-type (or nonanticipative) representation and Shepp-type representation. At least former seems to be closely related to the stochastic calculus and Itô's formula (see [5], Section 6.4.). Since the stochastic calculus in the multiparameter case is more complicated than in the one-parameter case, we ask ourselves if the representation theorems using stochastic integrals can yet be obtained. The answer is positive and it is due to the fact that actually the main tool used is the Gohberg-Krein factorization theorem (see [8]). Our study surveys different types of representations, canonical or non-canonical, for the equivalent Gaussian sheets. In the "fractional" case, we show a Hitsuda characterization theorem for Gaussian sheets equivalent to the fractional Brownian sheet only in the case when all parameters are bigger than $1 / 2$. The reason of the lack of a such characterization in the other cases consists in the structure of the linear Gaussian space generated by the fractional Brownian sheet, see [11] and [12] for the one-parameter case.

We organized our paper as follows. Section 2 contains the notation and preliminaries on Hilbert-Schmidt operators. In Section 3 we give a general (and rather abstract) result on the Gaussian equivalence. In Section 4 we characterize the Gaussian processes that are equivalent in law to the Brownian sheet and in Section 5 we consider the same topics for the fractional Brownian sheet. Section 6 is an application of our results to a special kind of stochastic equation with (fractional) Brownian sheet as noise term.

\section{Preliminaries and notation}

Let $\mathbf{t}=\left(t_{1}, t_{2}, \ldots, t_{d}\right)$ and $\mathbf{s}=\left(s_{1}, s_{2}, \ldots, s_{d}\right)$ be vectors in $\mathbb{R}^{d}$. By $\mathbf{s} \leq \mathbf{t}$ we mean the partial ordering $s_{i} \leq t_{i}$ for all $i=1,2, \ldots, d$. By $[0, \mathbf{t}]$ we denote $\left[0, t_{1}\right] \times\left[0, t_{2}\right] \times$ $\cdots \times\left[0, t_{d}\right]$. So, e.g.

$$
\int_{[0, \mathbf{t}]} f(\mathbf{s}) \mathrm{d} \mathbf{s}=\int_{0}^{t_{1}} \int_{0}^{t_{2}} \cdots \int_{0}^{t_{d}} f\left(s_{1}, s_{2}, \ldots, s_{d}\right) \mathrm{d} s_{d} \cdots \mathrm{d} s_{2} \mathrm{~d} s_{1} .
$$

Similarly, $[\mathbf{s}, \mathbf{t}]=\left[s_{1}, t_{1}\right] \times\left[s_{2}, t_{2}\right] \times \cdots \times\left[s_{d}, t_{d}\right]$.

A kernel $b:[0,1]^{2 d} \rightarrow \mathbb{R}$ is of Volterra type if $b(\mathbf{t}, \mathbf{s}) \neq 0$ implies $\mathbf{s} \leq \mathbf{t}$. Here $\mathbf{t}, \mathbf{s} \in \mathbb{R}^{d}$. The set of square integrable Volterra kernels is denoted by $L_{V}^{2}\left([0,1]^{2 d}\right)$. A kernel $b:[0,1]^{2 d} \rightarrow \mathbb{R}$ is symmetric if $b(\mathbf{t}, \mathbf{s})=b(\mathbf{s}, \mathbf{t})$. The set of symmetric square integrable kernels is denoted by $L_{S}^{2}\left([0,1]^{2 d}\right)$.

The tensor product $f \otimes g:[0,1]^{d+d^{\prime}} \rightarrow \mathbb{R}$ of functions $f:[0,1]^{d} \rightarrow \mathbb{R}$ and $g:$ $[0,1]^{d^{\prime}} \rightarrow \mathbb{R}$ is simply $(f \otimes g)(\mathbf{t}, \mathbf{s})=f(\mathbf{t}) g(\mathbf{s})$. The tensor product $\mathrm{K} \otimes \mathrm{H}$ of operators 
$\mathrm{K}$ and $\mathrm{H}$ is defined as $(\mathrm{K} \otimes \mathrm{H}) f(\mathbf{t}, \mathbf{s})=\left(\mathrm{K} g_{\mathbf{s}}\right)(\mathbf{t})$, where $g_{\mathbf{s}}=\mathbf{t} \mapsto \mathrm{H} f(\mathbf{t}, \cdot)(\mathbf{s})$. Multiple tensor products are defined through iteration.

Let $k$ be a kernel in $L^{2}\left([0,1]^{2 d}\right)$. The corresponding capital roman letter ( $\mathrm{K}$ in this case) denotes the Hilbert-Schmidt operator on $L^{2}\left([0,1]^{d}\right)$ induced by $k$ as

$$
\mathrm{K} f(\mathbf{t})=\int_{[0,1]^{d}} f(\mathbf{s}) k(\mathbf{t}, \mathbf{s}) \mathrm{d} \mathbf{s} .
$$

A Hilbert-Schmidt operator on $L^{2}\left([0,1]^{d}\right)$ always admits a kernel.

The trace of the operator $\mathrm{K}$ with kernel $k$ is simply

$$
\operatorname{Tr}(\mathrm{K})=\int_{[0,1]^{d}} k(\mathbf{t}, \mathbf{t}) \mathrm{d} \mathbf{t},
$$

if it exists, and in this case we say that $\mathrm{K}$ is of trace class.

If $\mathrm{K}$ and $\mathrm{H}$ are two Hilbert-Schmidt operators on $L^{2}\left([0,1]^{d}\right)$ with kernels $k$ and $h$ then in is easy the see that the composition HK is again a Hilbert-Schmidt operator. Indeed, the kernel associated to HK is the convolution

$$
(h \star k)(\mathbf{t}, \mathbf{s})=\int_{[0,1]^{d}} h(\mathbf{t}, \mathbf{u}) k(\mathbf{u}, \mathbf{s}) \mathrm{d} \mathbf{u} .
$$

The adjoint $\mathrm{K}^{*}$ of a Hilbert-Schmidt operator $\mathrm{K}$ is again a Hilbert-Schmidt operator, and its kernel $k^{*}$ is simply $k^{*}(\mathbf{t}, \mathbf{s})=k(\mathbf{s}, \mathbf{t})$.

Let $\mathcal{I}^{0}$ denote the identity operator (this notation is in accordance to the fractional integral operators to be defined later in Section 5) and let $\sigma(\mathrm{K})=\left\{\lambda_{1}, \lambda_{2}, \ldots\right\}$ denote the (countable) set of the eigenvalues of $\mathrm{K}$, or its spectrum. If $k \in L^{2}\left([0,1]^{2 d}\right)$ and $1 \notin \sigma(\mathrm{K})$ then there exists a unique kernel $k_{1} \in L^{2}\left([0,1]^{2 d}\right)$ called the resolvent of $k$, such that the corresponding operators satisfy

$$
\mathcal{I}^{0}-\mathrm{K}_{1}=\left(\mathcal{I}^{0}-\mathrm{K}\right)^{-1},
$$

or, equivalently,

$$
\mathrm{KK}_{1}=\mathrm{K}+\mathrm{K}_{1}=\mathrm{K}_{1} \mathrm{~K} \text {. }
$$

Note that we have $\left(\mathrm{K}_{1}\right)_{1}=\mathrm{K}$. The operator $\mathrm{K}_{1}$ is also called the resolvent of $\mathrm{K}$.

Remark 2.1 In the literature sometimes the operator $-\mathrm{K}_{1}$ (and correspondingly $\left.-k_{1}\right)$ is called the resolvent. Some authors even define the resolvent to be $\mathcal{I}^{0}-\mathrm{K}_{1}$ (which is no longer Hilbert-Schmidt).

The resolvent kernel $k_{1}$ may be represented as the Neumann series

$$
k_{1}(\mathbf{t}, \mathbf{s})=-\sum_{n=1}^{\infty} k^{\star n}(\mathbf{t}, \mathbf{s}),
$$


that is

$$
\mathrm{K}_{1}=-\sum_{n=1}^{\infty} \mathrm{K}^{n} .
$$

Also, if $k$ has the Mercer expansion along its eigenvalues and eigenfunctions

$$
k(\mathbf{t}, \mathbf{s})=\sum_{j=1}^{\infty} \lambda_{j} e_{j}(\mathbf{t}) e_{j}(\mathbf{s})
$$

then the resolvent kernel $k_{1}$ may be represented as

$$
k_{1}(\mathbf{t}, \mathbf{s})=-\sum_{j=1}^{\infty} \frac{\lambda_{j}}{1-\lambda_{j}} e_{j}(\mathbf{t}) e_{j}(\mathbf{s})
$$

The Carleman-Fredholm determinant of $\mathrm{K}$ is

$$
\operatorname{Det}_{2}(\mathrm{~K})=\prod_{j=1}^{\infty}\left(1-\lambda_{j}\right) e^{\lambda_{j}}
$$

It exists for any Hilbert-Schmidt operator since $\sum_{j=1}^{\infty}\left|\lambda_{j}\right|^{2}<\infty$.

A Hilbert-Schmidt operator $\mathrm{K}$ is of Volterra type if its kernel $k$ is a Volterra kernel. So, in this case we have

$$
\mathrm{K} f(\mathbf{t})=\int_{[0, \mathbf{t}]} f(\mathbf{s}) k(\mathbf{t}, \mathbf{s}) \mathrm{d} \mathbf{s} .
$$

The resolvent $\mathrm{K}_{1}$ of a Volterra operator $\mathrm{K}$ always exists since in this case the spectral radius $\sup \{|\lambda|: \lambda \in \sigma(\mathrm{K})\}$ is zero. The resolvent is also of Volterra type.

\section{General results on equivalence}

Let $X=\left(X_{\mathbf{t}}\right)_{\mathbf{t} \in[0,1]^{d}}$ be a centered multiparameter Gaussian process on the probability space $(\Omega, \mathcal{F}, \mathbf{P})$ with covariance

$$
\mathbf{E}\left[X_{\mathbf{t}} X_{\mathbf{s}}\right]=R_{\mathbf{P}}(\mathbf{s}, \mathbf{t}) \text { for every } \mathbf{s}, \mathbf{t} \in[0,1]^{d} .
$$

We assume that $X$ is mean square continuous and denote by $\mathcal{H}(X)$ the reproducing kernel Hilbert space (RKHS, for short) of the process $X$. That is, for every $f \in \mathcal{H}(X)$ we have

$$
\left\langle f, R_{\mathbf{P}}(\mathbf{t}, \cdot)\right\rangle_{\mathcal{H}(X)}=f(\mathbf{t})
$$

for every $\mathbf{t} \in[0,1]^{d}$. The Hilbert space $\mathcal{H}(X)$ is the closure of the subspace spanned by $R_{\mathbf{P}}(\mathbf{t}, \cdot), \mathbf{t} \in[0,1]^{d}$, with respect to the inner product $\langle\cdot, \cdot\rangle_{\mathcal{H}(X)}$. Note that the 
continuity of $R_{\mathbf{P}}$, i.e. the mean square continuity of $X$, implies that $\mathcal{H}(X)$ is separable.

For every $\mathbf{t} \in[0,1]^{d}$, let us denote by $L(X, \mathbf{t})$ the closed linear subspace of $L^{2}(\Omega)$ generated by the random variables $X_{\mathbf{u}}, \mathbf{u} \leq \mathbf{t}$, and let us consider the isometric isomorphism $\Phi$ from $L(X, \mathbf{1})$ onto the RKHS $\mathcal{H}(X)$ such that, for every $\mathbf{t} \in[0,1]^{d}$

$$
\Phi X_{\mathbf{t}}=R_{\mathbf{P}}(\mathbf{t}, \cdot)
$$

We will use the following notation

$$
F(\mathbf{t})=\{f \in \mathcal{H}(X) \text { such that } f(\mathbf{u})=0 \text { if } 0 \leq \mathbf{u} \leq \mathbf{t}\},
$$

and let $M(\mathbf{t})$ be the orthogonal complement of $F(\mathbf{t})$ in $\mathcal{H}(X)$. Clearly,

$$
M(\mathbf{t})=\left\{f \in \mathcal{H}(X) \text { such that } f(\mathbf{u})=0 \text { if } u_{i}>t_{i} \text { for some } i=1, \ldots, d\right\} .
$$

We have the following lemma.

Lemma 3.1 For every $\mathbf{t} \in[0,1]^{d}$ it holds that

$$
\Phi(L(X, \mathbf{t}))=M(\mathbf{t}) .
$$

Proof: It holds that

$f \in F(\mathbf{t})$ if and only if $\Phi^{-1} f$ belongs to the complement of $L(X, \mathbf{t})$ in $L(X, \mathbf{1})$.

Indeed, for all $\mathbf{u} \in[0, \mathbf{t}]$ we have

$$
0=f(\mathbf{u})=\left\langle f, R_{\mathbf{P}}(\mathbf{t}, \cdot)\right\rangle_{\mathcal{H}(X)} \Longleftrightarrow \mathbf{E}\left[\Phi^{-1} f \cdot X_{\mathbf{t}}\right]=0 .
$$

Let $\tilde{\mathbf{P}}$ be another probability measure on $(\Omega, \mathcal{F})$ such that $(X, \tilde{\mathbf{P}})$ is a centered Gaussian sheet with covariance $R_{\tilde{\mathbf{P}}}$. Using the same type of arguments as in [8] or [9], we can prove the following canonical nonanticipative representation.

Theorem 3.2 Every mean square continuous Gaussian sheet $(X, \tilde{\mathbf{P}})$ which is equivalent in law to a given Gaussian sheet $(X, \mathbf{P})$ admits a nonanticipative representation with respect to $(X, \mathbf{P})$. That is, there exists a centered Gaussian sheet $(Y, \mathbf{P})$ with covariance $R_{\tilde{\mathbf{P}}}$ such that $Y_{\mathbf{t}} \in L(X, \mathbf{t})$ for every $\mathbf{t} \in[0,1]^{d}$.

In the next section we show that in the case when the sheet $X$ above is equivalent in law to a Brownian sheet, Theorem 3.2 takes a less abstract form. 


\section{Gaussian processes equivalent in law to a Brownian sheet}

\subsection{Hitsuda representation}

Let $W=\left(W_{\mathbf{t}}\right)_{\mathbf{t} \in[0,1]^{d}}$ be a Brownian sheet on the probability space $(\Omega, \mathcal{F}, \mathbf{P})$. That means, $W$ is a centered multiparameter Gaussian process with the covariance

$$
\mathbf{E}\left[W_{\mathbf{t}} W_{\mathbf{s}}\right]=(\mathbf{s} \wedge \mathbf{t})=\prod_{i=1}^{d}\left(s_{i} \wedge t_{i}\right) .
$$

To prove the stochastic integral representation of processes, we will follow the lines of [8], Section 9.

Lemma 4.1 Let $b$ be a kernel in $L^{2}\left([0,1]^{2 d}\right)$ satisfying

$$
\int_{[0, \mathbf{t}]^{d}} b(\mathbf{u}, \mathbf{x}) f(\mathbf{u}) \mathrm{d} \mathbf{u} \neq 0 \quad \text { implies } \quad \mathbf{x} \leq \mathbf{u}
$$

for every $f \in L^{2}\left([0,1]^{d}\right)$. Then $b$ is a Volterra kernel, that is, $b \in L_{V}^{2}\left([0,1]^{2 d}\right)$.

Proof: Equation (4.1) implies that

$$
\int_{[0,1]^{2 d}} b(\mathbf{u}, \mathbf{x}) f(\mathbf{u}) 1_{[0, \mathbf{t}]}(\mathbf{u})\left(\prod_{i=1}^{d}\left(1-1_{\left[0, t_{i}\right]}\left(x_{i}\right)\right)\right) g(\mathbf{x}) \mathrm{d} \mathbf{u} \mathrm{d} \mathbf{x}=0
$$

for any $f, g \in L^{2}\left([0,1]^{d}\right)$.

Let us choose

$$
f(\mathbf{u})=1_{(a, b]}(\mathbf{u}) \text { and } g(\mathbf{x})=1_{(c, d]}(\mathbf{x})
$$

where for every $i=1 \ldots d$, we have

$$
\text { (either } a_{i} \geq d_{i} \text { or } b_{i} \leq c_{i} \text { ). }
$$

So we have, since $a_{i} \geq d_{i}$ or $b_{i} \leq c_{i}$,

$$
\int_{[0,1]^{2 d}} b(\mathbf{u}, \mathbf{x})\left(\prod_{i=1}^{d}\left(1-1_{\left[0, u_{i}\right]}\left(x_{i}\right)\right)\right) 1_{(a, b]}(\mathbf{u}) 1_{(c, d]}(\mathbf{x}) \mathrm{d} \mathbf{u} \mathrm{d} \mathbf{x}=0 .
$$

Since the family $\left\{1_{(a, b]}(x) 1_{(c, d]}(u), b \leq c\right.$ or $\left.a \geq d\right\}$ spans the space $L^{2}\left([0,1]^{2 d}\right)$, we obtain $b(\mathbf{u}, \mathbf{x})=0$ if there exists a coordinate $i$ with $x_{i}>u_{i}$.

The representation theorem for the Gaussian processes equivalent in law to the Brownian sheet is given below. 
Proposition 4.2 A centered Gaussian sheet $\tilde{W}=\left(\tilde{W}_{\mathbf{t}}\right)_{\mathbf{t} \in[0,1]^{d}}$ is equivalent in law to a Brownian sheet $W$ if and only if it admits the representation

$$
\tilde{W}_{\mathbf{t}}=W_{\mathbf{t}}-\int_{[0, \mathbf{t}]} \int_{[0, \mathbf{s}]} b(\mathbf{s}, \mathbf{u}) \mathrm{d} W_{\mathbf{u}} \mathrm{d} \mathbf{s}
$$

for some $b \in L_{V}^{2}\left([0,1]^{2 d}\right.$.

Proof: By Theorem 3.2, we have that for every $\mathbf{t} \in[0,1]^{d}, \tilde{W}_{\mathbf{t}} \in L(W, \mathbf{t}) \subset L(W, \mathbf{1})$. It follows from Kallianpur [8], pp. $225-233$ that $\Phi \tilde{W}_{\mathbf{t}}$ can be written as

$$
\Phi \tilde{W}_{\mathbf{t}}=\left(\mathcal{I}^{0}-\mathrm{B}\right) \Phi W_{\mathbf{t}}=\left(\mathcal{I}^{0}-\mathrm{B}\right) 1_{[0, \mathbf{t}]}(\cdot)
$$

where $\mathcal{I}^{0}$ (identity) and $\mathrm{B}$ are operators on $L^{2}\left([0,1]^{d}\right)$. Moreover, B is a Volterra operator. Therefore, we can write

$$
\begin{aligned}
\left(\mathcal{I}^{0}-\mathrm{B}\right) 1_{[0, \mathbf{t}]}(\cdot) & =1_{[0, \mathbf{t}]}(\cdot)-\int_{[0,1]^{d}} b(\mathbf{s}, \cdot) 1_{[0, \mathbf{t}]}(\mathbf{s}) \mathrm{d} \mathbf{s} \\
& =1_{[0, \mathbf{t}]}(\cdot)-\int_{[0, \mathbf{t}]} b(\mathbf{s}, \cdot) 1_{[0, \mathbf{t}]}(\mathbf{s}) \mathrm{d} \mathbf{s}
\end{aligned}
$$

where $b$ is the Volterra kernel corresponding to the Volterra operator B. (In fact, to be more precise it holds that

$$
\mathrm{B} P(\mathbf{t}) f=P(\mathbf{t}) \mathrm{B} P(\mathbf{t}) f
$$

for every $f \in L^{2}\left([0,1]^{d}\right)$ and $\mathbf{t} \in[0,1]^{d}$, where $P(\mathbf{t})$ denotes the orthoprojector on $L^{2}\left([0,1]^{d}\right)$ with range $M(\mathbf{t})$. Relation (4.3) and the fact that

$$
P(\mathbf{t}) f(\mathbf{s})=f(\mathbf{s}) 1_{[0, \mathbf{t}]}(\mathbf{s}) \quad \text { or } \quad \mathrm{B} P(\mathbf{t}) f(\mathbf{u})=\int_{[0, \mathbf{t}]} b(\mathbf{s}, \mathbf{u}) f(\mathbf{s}) \mathrm{d} \mathbf{s}
$$

implies that $b$ is a Volterra kernel.) The conclusion follows by Lemma 4.1.

The non-centered case is now obvious. Indeed, we know that admissible drifts are of the form

$$
A(\mathbf{t})=\int_{[0, \mathbf{t}]} a(\mathbf{s}) \mathrm{d} \mathbf{s} .
$$

for some $a \in L^{2}\left([0,1]^{d}\right)$. Also, by using the resolvents we may invert the relation (4.2). So, we have the following result:

Theorem 4.3 A Gaussian sheet $\tilde{W}$ is equivalent in law to the Brownian sheet $W$ if and only if there exists a Volterra kernel $b \in L_{V}^{2}\left([0,1]^{2 d}\right)$ and a function $a \in$ $L^{2}\left([0,1]^{d}\right)$ such that $\tilde{W}$ can be represented as

$$
\tilde{W}_{\mathbf{t}}=W_{\mathbf{t}}-\int_{[0, \mathbf{t}]} \int_{[0, \mathbf{s}]} b(\mathbf{s}, \mathbf{u}) \mathrm{d} W_{\mathbf{u}} \mathrm{d} \mathbf{s}-\int_{[0, \mathbf{t}]} a(\mathbf{s}) \mathrm{d} \mathbf{s} .
$$


The Volterra kernel $b$ and the function a are unique and the representation (4.4) is canonical in the sense that the sheets $\tilde{W}$ and $W$ generate the same filtration. Moreover, the Brownian sheet $W$ in (4.4) is constructed from the fractional one $\tilde{W}$ by

$$
W_{\mathbf{t}}=\tilde{W}_{\mathbf{t}}-\int_{[0, \mathbf{t}]} \int_{[0, \mathbf{s}]} b_{1}(\mathbf{s}, \mathbf{u}) \mathrm{d} \tilde{W}_{\mathbf{u}} \mathrm{d} \mathbf{s}-\int_{[0, \mathbf{t}]}\left(a(\mathbf{s})-\int_{[0, \mathbf{s}]} b_{1}(\mathbf{s}, \mathbf{u}) a(\mathbf{u}) \mathrm{d} u\right) \mathrm{d} \mathbf{s}
$$

where $b_{1} \in L_{V}^{2}\left([0,1]^{2 d}\right)$ is the resolvent of $b$.

The Hitsuda representation (4.4) is connected to the Girsanov representation of the density between $\mathbf{P}_{\tilde{W}}$ and $\mathbf{P}_{W}$ in the following way:

Theorem 4.4 The Radon-Nikodym derivative between the laws of $\tilde{W}$ and $W$ is

$$
\begin{aligned}
& \frac{\mathrm{d} \mathbf{P}_{\tilde{W}}}{\mathrm{~d} \mathbf{P}_{W}} \mid \mathcal{F}_{\mathbf{t}}=\exp \left\{\int_{[0, \mathbf{t}]}\left(\int_{[0, \mathbf{s}]} b(\mathbf{s}, \mathbf{u}) \mathrm{d} W_{\mathbf{u}}+a(\mathbf{s})\right) \mathrm{d} W_{\mathbf{s}}\right. \\
&\left.-\frac{1}{2} \int_{[0, \mathbf{t}]}\left(\int_{[0, \mathbf{s}]} b(\mathbf{s}, \mathbf{u}) \mathrm{d} W_{\mathbf{u}}+a(\mathbf{s})\right)^{2} \mathrm{~d} \mathbf{s}\right\} .
\end{aligned}
$$

In terms of $\tilde{W}$ the Radon-Nikodym derivative takes the form

$$
\begin{aligned}
\frac{\mathrm{d} \mathbf{P}_{\tilde{W}}}{\mathrm{~d} \mathbf{P}_{W}} \mid \mathcal{F}_{\mathbf{t}} & \exp \left\{\int_{[0, \mathbf{t}]}\left(\int_{[0, \mathbf{s}]} b_{1}(\mathbf{s}, \mathbf{u}) \mathrm{d} \tilde{W}_{\mathbf{u}}+a(\mathbf{s})-\int_{[0, \mathbf{s}]} b_{1}(\mathbf{s}, \mathbf{u}) a(\mathbf{u}) \mathrm{d} \mathbf{u}\right) \mathrm{d} \tilde{W}_{\mathbf{s}}\right. \\
& \left.\quad-\frac{1}{2} \int_{[0, \mathbf{t}]}\left(\int_{[0, \mathbf{s}]} b_{1}(\mathbf{s}, \mathbf{u}) \mathrm{d} \tilde{W}_{\mathbf{u}}+a(\mathbf{s})-\int_{[0, \mathbf{s}]} b_{1}(\mathbf{s}, \mathbf{u}) a(\mathbf{u}) \mathrm{d} \mathbf{u}\right)^{2} \mathrm{~d} \mathbf{s}\right\}
\end{aligned}
$$

Proof: The formula (4.6) is an immediate consequence of the multiparameter Girsanov formula (see [3] or [4]) and the isometry

$$
\mathbf{E}\left(\int_{[0, \mathbf{t}]} Y_{\mathbf{s}} \mathrm{d} W_{\mathbf{s}}\right)^{2}=\int_{[0, \mathbf{t}]} \mathbf{E} Y_{\mathbf{s}}^{2} \mathrm{~d} \mathbf{s} .
$$

The formula (4.7) follows from (4.6) by symmetry.

\subsection{Shepp representation}

The Shepp representation of Gaussian measures equivalent in law with the Wiener measure can be extended to the multiparameter case. 
Theorem 4.5 A Gaussian sheet $\tilde{W}$ is equivalent in law with the Brownian sheet $W$ if and only if there exists a kernel $k \in L_{S}^{2}\left([0,1]^{2 d}\right)$ such that $1 \notin \sigma(\mathrm{K})$ and a function $m \in L^{2}\left([0,1]^{d}\right)$ and

$$
\operatorname{Cov}\left(\tilde{W}_{\mathbf{t}}, \tilde{W}_{\mathbf{s}}\right)=\mathbf{s} \wedge \mathbf{t}-\int_{[0, \mathbf{t}]} \int_{[0, \mathbf{s}]} k(\mathbf{u}, \mathbf{v}) \mathrm{d} \mathbf{u} \mathrm{d} \mathbf{v}
$$

and

$$
\mathbf{E}\left[\tilde{W}_{\mathbf{t}}\right]=\int_{[0, \mathbf{t}]} m(\mathbf{u}) \mathrm{d} \mathbf{u} .
$$

The kernel $k$ is unique and symmetric and is given by

$$
k(\mathbf{s}, \mathbf{t})=-\frac{\partial^{2 d}}{\partial \mathbf{s} \partial \mathbf{t}} \mathbf{C o v}\left(\tilde{W}_{\mathbf{s}}, \tilde{W}_{\mathbf{t}}\right)
$$

for almost every $\mathbf{s}$ and $\mathbf{t}$. The function $m$ is also unique and it is given by

$$
m(\mathbf{t})=\frac{\partial^{d}}{\partial \mathbf{t}} \mathbf{E}\left[\tilde{W}_{t}\right]
$$

for almost all $\mathbf{t}$.

The Radon-Nikodym derivative $\frac{\mathrm{d} \mathbf{P}_{\tilde{W}}}{\mathrm{~d}_{W}}$ is given by

$$
\begin{gathered}
\frac{\mathrm{d} \mathbf{P}_{\tilde{W}}}{\mathrm{~d} \mathbf{P}_{W}}=\frac{1}{\sqrt{\operatorname{Det}_{2}(\mathrm{~K}) \operatorname{Tr}\left(\mathrm{K}_{1} \mathrm{~K}\right)}} \times \\
\exp \left\{-\frac{1}{2} \int_{[0,1]^{d}} \int_{[0,1]^{d}} k_{1}(\mathbf{t}, \mathbf{s}) \mathrm{d} \tilde{W}_{\mathbf{s}} \mathrm{d} \tilde{W}_{\mathbf{t}}+\int_{[0,1]^{d}} m(\mathbf{t}) \mathrm{d} \tilde{W}_{\mathbf{t}}+\frac{1}{2} \int_{[0,1]^{d}} m(\mathbf{t})^{2} \mathrm{~d} \mathbf{t}\right\} .
\end{gathered}
$$

Proof: The proof given in [14] can be applied exactly.

For the proof of next Corollary in the one-parameter case we refer to [2]. The multiparameter case may be proved similarly.

Corollary 4.6 Let $W$ a Brownian sheet and $Z$ be an independent Gaussian sheet. Then the the sheet $W+Z$ is equivalent to a Brownian sheet if and only if there exists $m \in L^{2}\left([0,1]^{d}\right)$ and $k \in L_{S}^{2}\left([0,1]^{2 d}\right)$ such that

$$
\mathbf{E}\left[Z_{\mathbf{t}}\right]=\int_{[0, \mathbf{t}]} m(\mathbf{s}) \mathrm{d} \mathbf{s},
$$

and

$$
\operatorname{Cov}\left(Z_{\mathbf{t}}, Z_{\mathbf{s}}\right)=\int_{[0, \mathbf{t}]} \int_{[0, \mathbf{s}]} k(\mathbf{u}, \mathbf{v}) \mathrm{d} \mathbf{u} \mathrm{d} \mathbf{v}
$$


Example 4.7 Let $H \in(0,1)$ and $K \in(0,1]$. Set

$$
R_{H, K}(s, t)=\frac{1}{2^{K}}\left(\left(t^{2 H}+s^{2 H}\right)^{K}-|t-s|^{2 H K}\right) .
$$

It has been proved in [7] that the function (4.11) is positive definite. So we may define a centered sheet $B=\left(B_{\mathbf{t}}^{\mathbf{H}, \mathbf{K}}\right)_{\mathbf{t} \in[0,1]^{d}}$ with the covariance

$$
R_{\mathbf{H}, \mathbf{K}}(\mathbf{t}, \mathbf{s})=\prod_{i=1}^{d} R_{H_{i}, K_{i}}\left(t_{i}, s_{i}\right) .
$$

According to Corollary 1, we have that $W+B^{\mathbf{H}, \mathbf{K}}$ is equivalent in law to a Brownian sheet if and only if

$$
\begin{aligned}
\frac{\partial^{2 d}}{\partial \mathbf{s} \partial \mathbf{t}} R_{\mathbf{H}, \mathbf{K}}(\mathbf{s}, \mathbf{t})= & \prod_{i=1}^{d} \frac{2 H_{i} K_{i}}{2^{K_{i}}}\left(2 H_{i}\left(K_{i}-1\right)\left(t_{i}^{2 H_{i}}+s_{i}^{2 H_{i}}\right)^{K_{i}-2} t_{i}^{2 H_{i}-1} s_{i}^{2 H_{i}-1}\right. \\
& \left.+\left(2 H_{i} K_{i}-1\right)\left(t_{i}-s_{i}\right)^{2 H_{i} K_{i}-2}\right)
\end{aligned}
$$

belongs to $L^{2}\left([0,1]^{2 d}\right)$. Majorizing $\left(t_{i}^{2 H}+s_{i}^{2 H}\right)^{K-2}$ by $\left(t_{i} s_{i}\right)^{H(K-2)}$ we obtain that this is true if and only if $H_{i} K_{i}>3 / 4$ for every $i$. In the case $K_{i}=1\left(B^{\mathbf{H}, 1}\right.$ is a standard fractional Brownian sheet, cf. next section) we have that $W+B^{\mathbf{H}, 1}$ is equivalent in law to a Brownian sheet if and only if $\min _{i=1, \ldots d} H_{i}>3 / 4$.

We end this section by comparing the Hitsuda and Shepp representations. The situation is similar to the one-parameter case studied by Cheridito [2]. Indeed, by comparing (4.7) and (4.10), we obtain the following.

Proposition 4.8 Let $b$ and $a$ be as in Theorem 4.4 and $k$ and $m$ as in Theorem 4.5. Then

$$
\begin{gathered}
a=m, \\
\mathbf{E} \exp \left\{-\frac{1}{2} \int_{[0,1]^{d}} \int_{[0,1]^{d}} k_{1}(\mathbf{t}, \mathbf{s}) \mathrm{d} \tilde{W}_{\mathbf{s}} \tilde{W}_{\mathbf{t}}\right\}=\exp \left\{\frac{1}{2} \int_{[0,1]^{d}} \int_{[0, \mathbf{t}]} b_{1}(\mathbf{t}, \mathbf{s}) \mathrm{d} \mathbf{s} \mathrm{d} \mathbf{t}\right\},
\end{gathered}
$$

and for the kernels $b$ and $k$ we have the relation

$$
k=b+b^{*}-b \star b^{*} .
$$

By symmetry, we have for the resolvent kernels $b_{1}$ and $k_{1}$ that

$$
k_{1}=b_{1}+b_{1}^{*}-b_{1} \star b_{1}^{*}
$$


Remark 4.9 In general, the equation (4.12) does not admit a unique solution in $b$. Indeed, given a kernel $b \in L^{2}([0,1])^{2 d}$ the sheet

$$
\tilde{W}_{\mathbf{t}}=W_{\mathbf{t}}-\int_{[0, \mathbf{t}]} \int_{[0,1]^{d}} b(\mathbf{t}, \mathbf{s}) \mathrm{d} W_{\mathbf{s}} \mathrm{d} \mathbf{t}
$$

is equivalent in law to a Brownian sheet and it $k$ in the Shepp representation in given by (4.12). If $b$ is a Volterra kernel, as it is in the Hitsuda representation, then (4.12) is the Gohberg-Krein factorization of $k, c f$. [8] where it is written in the symmetric form

$$
\mathcal{I}^{0}-\mathrm{K}=\left(\mathcal{I}^{0}-\mathrm{B}\right)\left(\mathcal{I}^{0}-\mathrm{B}^{*}\right)
$$

\section{Gaussian processes equivalent in law to fractional Brownian sheet}

\subsection{Wiener integrals and Hitsuda representation}

The fractional Brownian sheet $Z=\left(Z_{\mathbf{t}}^{\mathbf{H}}\right)_{\mathbf{t} \in[0,1]^{d}}$ with multiple Hurst index $\mathbf{H}=$ $\left(H_{1}, H_{2}, \ldots, H_{d}\right) \in(0,1)^{d}$ on $(\Omega, \mathcal{F}, \mathbf{P})$ is a centred Gaussian field with covariance function

$$
\begin{aligned}
R_{\mathbf{H}}(\mathbf{t}, \mathbf{s}) & =\prod_{i=1}^{d} R_{H_{i}}\left(t_{i}, s_{i}\right) \\
& =\prod_{i=1}^{d} \frac{1}{2}\left(t_{i}^{2 H_{i}}+s_{i}^{2 H_{i}}-\left|t_{i}-s_{i}\right|^{2 H_{i}}\right) .
\end{aligned}
$$

We want to derive a representation of the fractional Brownian sheet with respect to the ordinary one. To this end we recall some preliminaries of fractional calculus. For details we refer to [13].

Let $f$ be a function over the interval $[0,1]$ and $\alpha>0$. Then

$$
\mathcal{I}_{ \pm}^{\alpha} f(t)=\frac{1}{\Gamma(\alpha)} \int_{0}^{1} \frac{f(s)}{(t-s)_{ \pm}^{1-\alpha}} \mathrm{d} s
$$

are the Riemann-Liouville fractional integrals of order $\alpha$. For $\alpha \in(0,1)$,

$$
\mathcal{D}_{ \pm}^{\alpha} f(t)=\frac{ \pm 1}{\Gamma(1-\alpha)} \frac{\mathrm{d}}{\mathrm{d} t} \int_{0}^{1} \frac{f(s)}{(t-s)_{ \pm}^{\alpha}} \mathrm{d} s
$$

are the Riemann-Liouville fractional derivatives of order $\alpha ; \mathcal{I}_{ \pm}^{0}$ and $\mathcal{D}_{ \pm}^{0}$ are identity operators. 
If one ignores the troubles concerning divergent integrals and formally changes the order of differentiation and integration one obtains

$$
\mathcal{I}_{ \pm}^{-\alpha}=\mathcal{D}_{ \pm}^{\alpha}
$$

We shall take the above as the definition for fractional integral of negative order.

Remark The operators $\mathcal{I}_{ \pm}^{\alpha}$ are not Hilbert-Schmidt, that is, they do not admit kernels; Hence the use of calligraphic letters.

By [13], Theorem 2.5, the composition formula

$$
\mathcal{I}_{ \pm}^{\alpha} \mathcal{I}_{ \pm}^{\beta} f=\mathcal{I}_{ \pm}^{\alpha+\beta} f
$$

is valid in any of the following cases.

(i) $\beta \geq 0, \alpha+\beta \geq 0$ and $f \in L^{1}([0,1])$,

(ii) $\beta \leq 0, \alpha \geq 0$ and $f \in \mathcal{I}_{ \pm}^{-\beta} L^{1}([0,1])$,

(iii) $\alpha \leq 0, \alpha+\beta \leq 0$ and $f \in \mathcal{I}_{ \pm}^{-\alpha-\beta} L^{1}([0,1])$.

Let us now briefly consider Wiener integrals with respect to fractional Brownian sheet. Details for the one-parameter case may be found in Pipiras and Taqqu [11, 12].

Let us introduce operators

$$
\begin{aligned}
\mathcal{K}_{\beta} f(t) & =c_{\beta} t^{\frac{1}{2}-\beta}\left(\mathcal{I}_{-}^{\beta-\frac{1}{2}} s^{\beta-\frac{1}{2}} f(s)\right)(t), \\
\mathcal{K}_{\beta}^{-1} f(t) & =\frac{1}{c_{\beta}} t^{\frac{1}{2}-\beta}\left(\mathcal{I}_{-}^{\frac{1}{2}-\beta} s^{\beta-\frac{1}{2}} f(s)\right)(t),
\end{aligned}
$$

where

$$
c_{\beta}=\sqrt{\frac{2 \beta\left(\beta-\frac{1}{2}\right) \Gamma\left(\beta-\frac{1}{2}\right)^{2}}{\mathrm{~B}\left(\beta-\frac{1}{2}, 2-2 \beta\right)}} .
$$

Now, for $i=1,2, \ldots, d$ we have

$$
R_{H_{i}}\left(t_{i}, s_{i}\right)=\left\langle\mathcal{K}_{H_{i}} \mathbf{1}_{\left[0, t_{i}\right]}, \mathcal{K}_{H_{i}} \mathbf{1}_{\left[0, s_{i}\right]}\right\rangle_{L^{2}([0,1])} .
$$

So, if we set

$$
\mathcal{K}_{\mathbf{H}}=\mathcal{K}_{H_{1}} \otimes \mathcal{K}_{H_{2}} \otimes \cdots \otimes \mathcal{K}_{H_{d}}
$$


we see by using the Fubini theorem that

$$
\begin{aligned}
R_{\mathbf{H}}(\mathbf{t}, \mathbf{s}) & =\prod_{i=1}^{d} R_{H_{i}}\left(t_{i}, s_{i}\right) \\
& =\prod_{i=1}^{d}\left\langle\mathcal{K}_{H_{i}} \mathbf{1}_{\left[0, t_{i}\right]}, \mathcal{K}_{H_{i}} \mathbf{1}_{\left[0, s_{i}\right]}\right\rangle_{L^{2}([0,1])} \\
& =\int_{[0,1]^{d}} \prod_{i=1}^{d} \mathcal{K}_{H_{i}} \mathbf{1}_{\left[0, t_{i}\right]}\left(u_{i}\right) \cdot \prod_{i=1}^{d} \mathcal{K}_{H_{1}} \mathbf{1}_{\left[0, s_{i}\right]}\left(u_{i}\right) \mathrm{d} \mathbf{u} \\
& =\left\langle\mathcal{K}_{\mathbf{H}} \mathbf{1}_{[0, \mathbf{t}]}, \mathcal{K}_{\mathbf{H}} \mathbf{1}_{[0, \mathbf{s}]}\right\rangle_{L^{2}\left([0,1]^{d}\right)} .
\end{aligned}
$$

Consequently, we have the representation

$$
Z_{\mathbf{t}}=\int_{[0, \mathbf{t}]} \mathcal{K}_{\mathbf{H}} \mathbf{1}_{[0, \mathbf{t}]}(\mathbf{s}) \mathrm{d} W_{\mathbf{s}} .
$$

Also, the operator $\mathcal{K}_{\mathbf{H}}$ is injective and has simple functions in its image. Thus we have a reverse representation

$$
W_{\mathbf{t}}=\int_{[0, \mathbf{t}]} \mathcal{K}_{\mathbf{H}}^{-1} \mathbf{1}_{[0, \mathbf{t}]}(\mathbf{s}) \mathrm{d} Z_{\mathbf{s}} .
$$

Actually, the inverse operator $\mathcal{K}_{\mathbf{H}}^{-1}$ is given as

$$
\mathcal{K}_{H}^{-1}=\mathcal{K}_{H_{1}}^{-1} \otimes \mathcal{K}_{H_{2}}^{-1} \otimes \cdots \otimes \mathcal{K}_{H_{d}}^{-1} .
$$

Also, note that by Proposition 3.4. of [17] the representations (5.2) and (5.3) may be understood pathwise.

We drop the Hurst indices in the notations now.

Since the integral with respect to the Brownian sheet is defined for any $f \in$ $L^{2}\left([0,1]^{d}\right)$ we may define:

Definition 5.1 Set

$$
\Lambda=\left\{f: \mathcal{K} f \in L^{2}\left([0,1]^{d}\right)\right\} .
$$

The Wiener integral of $f \in \Lambda$ with respect to fractional Brownian sheet is

$$
\int_{[0,1]^{d}} f(\mathbf{t}) \mathrm{d} Z_{\mathbf{t}}=\int_{[0,1]^{d}} \mathcal{K} f(\mathbf{t}) \mathrm{d} W_{\mathbf{t}} .
$$

The integral in Definition 5.1 can be considered as a limit of elementary functions. Indeed, by Theorems 4.1 and 4.2 of [12] the set $\Lambda$ is a Pre-Hilbert space with

$$
\langle f, g\rangle_{\Lambda}=\langle\mathcal{K} f, \mathcal{K} g\rangle_{L^{2}\left([0,1]^{d}\right)}
$$


and the set of step functions is dense in $\Lambda$.

Obviously, if $f$ is a step function then $\mathcal{K} \mathcal{K}^{-1} f=f=\mathcal{K}^{-1} \mathcal{K} f$. Also, from the composition formula (5.1) and tensorization it follows that for any $f \in L^{2}\left([0,1]^{d}\right)$ we have $\mathcal{K}^{-1} \mathcal{K} f=f$ if $\min _{i \leq d} H_{i} \geq \frac{1}{2}$ and $\mathcal{K} \mathcal{K}^{-1} f=f$ if $\max _{i \leq d} H_{i} \leq \frac{1}{2}$. However, we have the following.

Lemma 5.2 Let $\max _{i \leq d} H_{i}>\frac{1}{2}$. Then there exist functions $f \in L^{2}\left([0,1]^{d}\right)$ such that the equation

$$
\mathcal{K} g=f
$$

has no solution in $g$.

The reason for the lack of solutions in (5.4) is that for $H_{i}>1 / 2$ the operator $\mathcal{K}$ is a weighted fractional integral operator on coordinate $i$. So, one can partially differentiate $f$ fractionally $\left(\mathcal{K}^{-1}\right.$ is a weighted fractional differential operator on coordinate $i$ ). However, there are "arbitrarily non-smooth" functions in $L^{2}\left([0,1]^{d}\right)$. An example of such a function is

$$
f(\mathbf{t})=\prod_{i=1}^{d} t_{i}^{\frac{1}{2}-H_{i}} \psi_{i}\left(t_{i}\right)
$$

where $\psi_{i}$ is the real part of the Weierstrass function

$$
\psi_{i}^{*}(t)=\sum_{n=1}^{\infty} b^{-p_{i} n} e^{i b^{n} t},
$$

$b>1$ and $p_{i} \in\left(0, H_{i}-1 / 2\right)$. For the rigorous proof of Lemma in the one-parameter case 5.2 see [12], Lemma 5.3.

The reproducing kernel Hilbert space $\mathcal{H}(Z)$ of the fractional Brownian sheet is easy to identify.

Proposition 5.3 A function $f \in \mathcal{H}(Z)$ if and only if it can be represented as

$$
f(\mathbf{t})=\int_{[0, \mathbf{t}]} \mathcal{K} \mathbf{1}_{[0, \mathbf{t}]}(\mathbf{s}) \tilde{f}(\mathbf{s}) \mathrm{d} \mathbf{s}
$$

for some $\tilde{f} \in L^{2}\left([0,1]^{d}\right)$. The scalar product $\langle\cdot, \cdot\rangle_{\mathcal{H}(Z)}$ on $\mathcal{H}(Z)$ is given by

$$
\langle f, g\rangle_{\mathcal{H}}=\langle\tilde{f}, \tilde{g}\rangle_{L^{2}\left([0,1]^{d}\right)} .
$$

Moreover, as a vector space

$$
\mathcal{H}(Z)=\left(\bigotimes_{i=1}^{d} \mathcal{I}_{+}^{H_{i}+\frac{1}{2}}\right)\left(L^{2}\left([0,1]^{d}\right)\right) .
$$


Proof: The forms (5.6) and (5.7) follow immediately from the relation (5.2). The equality (5.8) follows from the isomorphism in [13], p. 187.

Recall that $\Lambda$ is isometric to $\mathcal{H}(Z)$. It is desirable that it is also isometric to the linear space $L(Z, \mathbf{t})$, i.e. one wants, as in the case of the Brownian sheet, to identify any $F \in L(Z, \mathbf{t})$ with a single function $f \in \Lambda$ so that

$$
F=\int_{[0,1]^{d}} f(\mathbf{t}) \mathrm{d} Z_{\mathbf{t}}
$$

Obviously, this is possible if and only if $\Lambda$ is complete. Otherwise $\Lambda$ is isometric to a proper subspace of $L(Z, \mathbf{t})$. Indeed, if $H_{i} \leq 1 / 2$, for all $i=1,2, \ldots, d$ then

$$
\Lambda=\left\{\mathcal{K}^{-1} f: f \in L^{2}\left([0,1]^{d}\right)\right\} .
$$

In particular, $\Lambda$ is complete and hence isometric to $L(Z, \mathbf{t})$. On the other hand, if $H_{i}>1 / 2$ for some $i$ then Lemma 5.2 implies that $\Lambda$ is not complete and hence isometric to a proper subspace of $L(Z, \mathbf{t})$.

Let us summarize the discussion above as a lemma:

Lemma 5.4 For $H_{i} \leq \frac{1}{2}$ for all $i=1,2, \ldots, d$ then the equality

$$
\int_{[0,1]^{d}} g(\mathbf{t}) \mathrm{d} W_{\mathbf{t}}=\int_{[0,1]^{d}} \mathcal{K}^{-1} g(\mathbf{t}) \mathrm{d} Z_{\mathbf{t}}
$$

holds for any $g \in L^{2}\left([0,1]^{d}\right)$. If $H_{i}$ exceeds $\frac{1}{2}$ for some $i=1,2, \ldots, d$ then the equality (5.9) holds only for $g \in \mathcal{K}(\Lambda)$ and the inclusion $\mathcal{K}(\Lambda) \subset L^{2}\left([0,1]^{d}\right)$ is strict.

As the fractional Brownian sheet $Z$ is mean square continuous we know now by the generalisation of the Kallianpur-Oodaira representation that any Gaussian sheet $\tilde{Z}$ that is equivalent in law to it can be represented canonically in terms of $Z$ in the sense that

$$
\tilde{Z}_{\mathbf{t}} \in L(Z, \mathbf{t}) \text {. }
$$

In the case of ordinary Brownian sheet $W$ the elements in the linear space $L(W, \mathbf{t})$ were Wiener integrals of the form

$$
\int_{[0, \mathbf{t}]} f(\mathbf{s}) \mathrm{d} W_{\mathbf{s}}
$$

for some $f \in L^{2}\left([0,1]^{d}\right)$. In the case of fractional Brownian sheet this is not generally true, as Lemma 5.2 suggests. Therefore, in general we do not have the Hitsuda representation for the fractional Brownian sheet as we have for the ordinary Brownian sheet. Indeed, the situation is similar to the one-parameter case studied in [15]. 
Let us introduce operators acting on Volterra kernels:

$$
\begin{aligned}
\mathcal{V}^{-1} b(\mathbf{t}, \mathbf{s}) & =\int_{[0,1]^{d}} \mathcal{K} \mathbf{1}_{[0, \mathbf{t}]}(\mathbf{u}) \mathcal{K}^{-1} b(\mathbf{u}, \cdot)(\mathbf{s}) \mathrm{d} \mathbf{u}, \\
\mathcal{V} b(\mathbf{t}, \mathbf{s}) & =\int_{[0,1]^{d}} \mathcal{K}^{-1} \mathbf{1}_{[0, \mathbf{t}]}(\mathbf{s}) \mathcal{K} b(\mathbf{u}, \cdot)(\mathbf{s}) \mathrm{d} \mathbf{u} .
\end{aligned}
$$

Note that $\mathcal{V}^{-1}$ is defined for kernels $b \in L_{V}^{2}\left([0,1]^{2 d}\right)$ with the property that $b(\mathbf{t}, \cdot) \in \Lambda$ for all $\mathbf{t} \in[0,1]^{d}$. If $b \in \mathcal{V}^{-1}\left(L_{V}^{2}\left([0,1]^{2 d}\right)\right)$ then $\mathcal{V} b$ exists. In particular, $b=\mathcal{V V}^{-1} b$.

Theorem 5.5 Let $Z$ be a fractional Brownian sheet with index $\mathbf{H} \in(0,1)^{d}$.

(i) A Gaussian sheet $\tilde{Z}$ given by the equation

$$
\tilde{Z}_{\mathbf{t}}=Z_{\mathbf{t}}-\int_{[0, \mathbf{t}]^{d}} f(\mathbf{t}, \mathbf{s}) \mathrm{d} Z_{\mathbf{s}}-A(\mathbf{t})
$$

is equivalent in law to $Z$ if and only if

$$
\begin{aligned}
& f \in \mathcal{V}^{-1}\left(L^{2}\left([0,1]^{2 d}\right)\right), \\
& A \in\left(\bigotimes_{i=1}^{d} I_{+}^{H_{i}+\frac{1}{2}}\right)\left(L^{2}\left([0,1]^{d}\right)\right) .
\end{aligned}
$$

Moreover, the fractional Brownian sheet $Z$ is constructed from $\tilde{Z}$ by

$$
Z_{\mathbf{t}}=\tilde{Z}_{\mathbf{t}}-\int_{[0, \mathbf{t}]} \mathcal{V}^{-1}(\mathcal{V} f)_{1}(\mathbf{t}, \mathbf{s}) \mathrm{d} \tilde{Z}_{\mathbf{s}}-A(\mathbf{t})+\int_{[0, \mathbf{t}]} \mathcal{V}^{-1}(\mathcal{V} f)_{1}(\mathbf{t}, \mathbf{s}) \mathrm{d} A(\mathbf{s})
$$

where $(\mathcal{V} f)_{1}$ is the resolvent of $\mathcal{V} f$.

(ii) If $\max _{i \leq d} H_{i} \leq 1 / 2$ then a Gaussian sheet is equivalent in law to a fractional Brownian sheet if and only if it is given by (5.10). If $\max _{i \leq d} H_{i}>1 / 2$ then there are Gaussian sheets equivalent in law to the fractional Brownian sheet that do not admit the representation (5.10).

Remark 5.6 The terms in (5.10) are of different form than in (4.5), i.e. they are not differentiable. The reason for this lies in the structure of the reproducing kernel Hilbert space of fractional Brownian sheet, cf. Proposition 5.3.

We have the following Kallianpur-Oodaira representations.

Proposition 5.7 A Gaussian sheet $\tilde{Z}$ is equivalent in law to a fractional Brownian sheet $Z$ if and only if it can be represented as

$$
\tilde{Z}_{\mathbf{t}}=Z_{\mathbf{t}}-\int_{[0, \mathbf{t}]} \mathcal{K} \mathbf{1}_{[0, \mathbf{t}]}(\mathbf{s}) \int_{[0, \mathbf{s}]} b(\mathbf{s}, \mathbf{u}) \mathrm{d} W_{\mathbf{u}} \mathrm{d} \mathbf{s}-\int_{[0, \mathbf{t}]} \mathcal{K} \mathbf{1}_{[0, \mathbf{t}]}(\mathbf{s}) a(\mathbf{s}) \mathrm{d} \mathbf{s}
$$


where $W$ is a Brownian sheet constructed from $Z$ by (5.3), $a \in L^{2}\left([0,1]^{d}\right)$ and $b \in L_{V}^{2}\left([0,1]^{2 d}\right)$. Moreover, the fractional Brownian sheet $Z$ is constructed from $\tilde{Z}$ by

$$
\begin{aligned}
Z_{\mathbf{t}}= & \tilde{Z}_{\mathbf{t}}-\int_{[0, \mathbf{t}]} \mathcal{K} \mathbf{1}_{[0, \mathbf{t}]}(\mathbf{s}) \int_{[0, \mathbf{s}]} b_{1}(\mathbf{s}, \mathbf{u}) \mathrm{d} \tilde{W}_{\mathbf{u}} \mathrm{d} \mathbf{s} \\
& +\int_{[0, \mathbf{t}]} \mathcal{K} \mathbf{1}_{[0, \mathbf{t}]}(\mathbf{s})\left\{a(\mathbf{s})-\int_{[0, \mathbf{s}]} b_{1}(\mathbf{s}, \mathbf{u}) a(\mathbf{u}) \mathrm{d} \mathbf{u}\right\} \mathrm{d} \mathbf{s}
\end{aligned}
$$

where $\tilde{W}$ is constructed from $\tilde{Z}$ by

$$
\tilde{W}_{\mathbf{t}}=\int_{[0, \mathbf{t}]} \mathcal{K}^{-1} \mathbf{1}_{[0, \mathbf{t}]}(\mathbf{s}) \mathrm{d} \tilde{Z}_{\mathbf{s}}
$$

and $b_{1} \in L_{V}^{2}\left([0,1]^{d}\right)$ is the resolvent of $b$.

By using Proposition 5.3 we can rephrase (5.12) in Proposition 5.7.

Proposition 5.8 A Gaussian sheet $\tilde{Z}$ is equivalent in law to a fractional Brownian sheet $Z$ with index $\mathbf{H}$ if and only if it can be represented as

$$
\tilde{Z}_{\mathbf{t}}=Z_{\mathbf{t}}-\int_{[0, \mathbf{t}]} f(\mathbf{t}, \mathbf{s}) \mathrm{d} W_{\mathbf{s}}-A(\mathbf{t})
$$

where $W$ is a Brownian sheet constructed from $Z$ by (5.3),

$$
A=\left(\bigotimes_{i=1}^{d} \mathcal{I}_{+}^{H_{i}+\frac{1}{2}}\right) a
$$

for some $a \in L^{2}\left([0,1]^{d}\right)$ and for all $\mathbf{s} \in[0,1]^{d}$

$$
f(\cdot, \mathbf{s})=\left(\bigotimes_{i=1}^{d} \mathcal{I}_{+}^{H_{i}+\frac{1}{2}}\right) b(\cdot, \mathbf{s})
$$

for some $b \in L_{V}^{2}\left([0,1]^{2 d}\right)$.

The proofs of Theorem 5.5 and Propositions 5.7 and 5.8 are easy search-andreplace modifications of the corresponding one-parameter case studied in [15]. It would be too boring to reproduce them here.

The obvious difference between the representations (4.4) and (5.12) (or (5.13)) is that in the latter one needs to construct a Brownian sheet from $Z$ in order to represent $\tilde{Z}$. Nevertheless, (5.12) and (5.13) are Kallianpur-Oodaira representations of $\tilde{Z}$ with respect to $Z$, since obviously $\tilde{Z}_{\mathbf{t}} \in L(Z, \mathbf{t})$. In the case $\max _{i \leq d} H_{i} \leq 1 / 2$ (and in this case only) we can use Lemma 5.4 to obtain a representation without the auxiliary Brownian sheet. This is the essence of Theorem 5.5. 
Remark 5.9 Note that since each one of the variables $W_{\mathbf{t}}, \tilde{W}_{\mathbf{t}}, Z_{\mathbf{t}}$, and $\tilde{Z}_{\mathbf{t}}$ can be constructed from any of the corresponding sheets by using only the information upto multi-time $\mathbf{t}$ the corresponding filtrations coincide: $\mathbf{F}^{W}=\mathbf{F}^{\tilde{W}}=\mathbf{F}^{Z}=\mathbf{F}^{\tilde{Z}}$.

\subsection{Double Wiener integrals and Girsanov representation}

Suppose that a Gaussian sheet $\tilde{Z}$ is equivalent in law to a fractional Brownian sheet $Z$. If $\tilde{Z}$ is given by (5.12) then the density is of course given by (4.6):

$$
\begin{aligned}
\frac{\mathrm{d} \mathbf{P}_{\tilde{Z}}}{\mathrm{~d} \mathbf{P}_{Z}} \mid \mathcal{F}_{\mathbf{t}}=\exp ( & \int_{[0, \mathbf{t}]}\left(\int_{[0, \mathbf{s}]} b(\mathbf{s}, \mathbf{u}) \mathrm{d} W_{\mathbf{u}}+a(\mathbf{s})\right) \mathrm{d} W_{\mathbf{s}} \\
& \left.-\frac{1}{2} \int_{[0, t]}\left(\int_{[0, \mathbf{s}]} b(\mathbf{s}, \mathbf{u}) \mathrm{d} W_{\mathbf{u}}+a(\mathbf{s})\right)^{2} \mathrm{~d} \mathbf{s}\right)
\end{aligned}
$$

where the Brownian sheet $W$ is constructed from from the fractional one by (5.3). If $\tilde{Z}$ is given by (5.10) then we know that the inner integral in (4.6) can be represented in terms of $Z$. However, to consider the outer integral we have to define what we mean by a stochastic or double Wiener integral with respect to fractional Brownian sheet.

In one-parameter case in [10] the authors defined

$$
\int_{0}^{1} \int_{0}^{1} b(t, s) \mathrm{d} Z_{s} \mathrm{~d} Z_{t}=\int_{0}^{1} \int_{0}^{1} \mathcal{K}_{2} b(t, s) \mathrm{d} W_{s} \mathrm{~d} W_{t}
$$

where $\mathcal{K}_{2}=\mathcal{K} \otimes \mathcal{K}$ and the right hand side of (5.14) is a double Wiener integral in the sense of Itô. In the multiparameter case the analogous definition is obvious.

Definition 5.10 Set $\mathcal{K}_{2}=\mathcal{K} \otimes \mathcal{K}$ and let

$$
\Lambda_{2}=\left\{b: \mathcal{K}_{2} b \in L^{2}\left([0,1]^{2 d}\right)\right\} .
$$

The double Wiener integral of $f \in \Lambda_{2}$ with respect to fractional Brownian sheet is

$$
\int_{[0,1]^{d}} \int_{[0,1]^{d}} f(\mathbf{t}, \mathbf{s}) \mathrm{d} Z_{\mathbf{s}} \mathrm{d} Z_{\mathbf{t}}=\int_{[0,1]^{d}} \int_{[0,1]^{d}} \mathcal{K}_{2} f(\mathbf{t}, \mathbf{s}) \mathrm{d} W_{\mathbf{s}} \mathrm{d} W_{\mathbf{t}} .
$$

The situation is now similar to the single Wiener integral case: If $\max _{i \leq d} H_{i} \leq 1 / 2$ then $\Lambda_{2}$ is complete and otherwise it is not. Indeed, in this case

$$
\Lambda_{2}=\left\{\mathcal{K}_{2}^{-1} f: f \in L^{2}\left([0,1]^{2 d}\right)\right\},
$$

where $\mathcal{K}_{2}^{-1}=\mathcal{K}^{-1} \otimes \mathcal{K}^{-1}$. Thus: 
Theorem 5.11 If $\max _{i \leq d} H \leq 1 / 2$ then any sheet $\tilde{Z}$ that is equivalent to the fractional Brownian sheet is given by (5.10) and we have

$$
\begin{aligned}
\frac{\mathrm{d} \mathbf{P}_{\tilde{Z}}}{\mathrm{~d} \mathbf{P}_{Z}} \mid \mathcal{F}_{\mathbf{t}}=\exp \left(\int_{[0, \mathbf{t}]} \int_{[0, \mathbf{t}]} \mathcal{K}_{2}^{-1} b(\mathbf{s}, \mathbf{u}) \mathrm{d} Z_{\mathbf{u}} \mathrm{d} Z_{\mathbf{s}}+\int_{0, \mathbf{t}]} \mathcal{K}^{-1} a(\mathbf{s}) \mathrm{d} Z_{\mathbf{s}}\right. \\
\left.-\frac{1}{2} \int_{[0, \mathbf{t}]}\left(\int_{[0, \mathbf{t}]} \mathcal{K}^{-1} b(\mathbf{s}, \cdot)(\mathbf{u}) \mathrm{d} Z_{\mathbf{u}}+\mathcal{K}^{-1} a(\mathbf{s})\right)^{2} \mathrm{~d} \mathbf{s}\right)
\end{aligned}
$$

where $b=\mathcal{V} f$ and $A=\int_{[0, \cdot]} \mathcal{K} \mathbf{1}_{[0, \cdot]}(\mathbf{s}) a(\mathbf{s}) \mathrm{d} \mathbf{s}$.

If $\max _{i \leq d} H_{i}>1 / 2$ then there are sheets $\tilde{Z}$ that are equivalent to the fractional Brownian sheet $Z$ that do not admit the representation (5.15) even if $\tilde{Z}$ admits the representation (5.10).

The first claim of Theorem 5.11 is obvious. To see the latter claim take $g=$ $g_{1} \otimes g_{2} \in L^{2}\left([0,1]^{2 d}\right)$ such that $g_{2}$ is smooth but $g_{1}$ is not. Then apply Lemma 5.2.

\subsection{Shepp representation}

The Shepp representation of a Gaussian sheet $\tilde{Z}$ that is equivalent in law to the fractional Brownian sheet $Z$ is easily obtained from the Shepp representation for the Brownian sheet and the connection (5.2).

Theorem 5.12 A Gaussian sheet $\tilde{Z}$ is equivalent in law to a fractional Brownian sheet $Z$ if and only if there exists a kernel $k \in L_{S}^{2}\left([0,1]^{2 d}\right)$ such that $1 \notin \sigma(\mathrm{K})$ and a function $m \in L^{2}\left([0,1]^{d}\right)$ such that

$$
\operatorname{Cov}\left(\tilde{Z}_{\mathbf{t}}, \tilde{Z}_{\mathbf{s}}\right)=\operatorname{Cov}\left(Z_{\mathbf{t}}, Z_{\mathbf{s}}\right)-\left(\bigotimes_{i=1}^{d} \mathcal{I}_{+}^{H_{i}+\frac{1}{2}} \otimes \bigotimes_{i=1}^{d} \mathcal{I}_{+}^{H_{i}+\frac{1}{2}}\right) k(\mathbf{t}, \mathbf{s})
$$

and

$$
\mathbf{E}\left[\tilde{Z}_{\mathbf{t}}\right]=\left(\bigotimes_{i=1}^{d} \mathcal{I}_{+}^{H_{i}+\frac{1}{2}}\right) m(\mathbf{t})
$$

The kernel $k$ and the function $m$ are unique. Indeed, they may be obtained, almost everywhere, from the covariance and mean functions by applying the inverses of the tensorized operators above.

The Radon-Nikodym derivative $\mathrm{d} \mathbf{P}_{\tilde{Z}} / \mathrm{d} \mathbf{P}_{Z}$ is just (4.10), where $\tilde{W}$ is constructed from $\tilde{Z}$ by

$$
\tilde{W}_{\mathbf{t}}=\int_{[0, \mathbf{t}]} \mathcal{K}^{-1} \mathbf{1}_{[0, \mathbf{t}]}(\mathbf{s}) \mathrm{d} \tilde{Z}_{\mathbf{s}}
$$


If $\max _{i \leq d} H_{i} \leq 1 / 2$, and in this case only, we may write (4.10) in terms of $\tilde{Z}$ :

$$
\begin{gathered}
\frac{\mathrm{d} \mathbf{P}_{\tilde{Z}}}{\mathrm{~d} \mathbf{P}_{Z}}=\frac{1}{\sqrt{\operatorname{Det}_{2}(\mathrm{~K}) \operatorname{Tr}\left(\mathrm{K}_{1} \mathrm{~K}\right)}} \times \\
\exp \left\{-\frac{1}{2} \int_{[0,1]^{d}} \int_{[0,1]^{d}} \mathcal{K}_{2}^{-1} k_{1}(\mathbf{t}, \mathbf{s}) \mathrm{d} \tilde{Z}_{\mathbf{s}} \mathrm{d} \tilde{Z}_{\mathbf{t}}+\int_{[0,1]^{d}} \mathcal{K}^{-1} m(\mathbf{t}) \mathrm{d} \tilde{Z}_{\mathbf{t}}+\frac{1}{2} \int_{[0,1]^{d}} m(\mathbf{t})^{2} \mathrm{~d} \mathbf{t}\right\} .
\end{gathered}
$$

Proof: By Theorem 5.5 we already know the form of the mean function. Let us then consider the covariance. From (5.12) we see that

$$
\operatorname{Cov}\left(\tilde{Z}_{\mathbf{t}}, \tilde{Z}_{\mathbf{s}}\right)=\operatorname{Cov}\left(Z_{\mathbf{t}}, Z_{\mathbf{s}}\right)-\int_{[0, \mathbf{t}]} \int_{[0, \mathbf{s}]} \mathcal{K} \mathbf{1}_{[0, \mathbf{t}]}(\mathbf{u}) \mathcal{K} \mathbf{1}_{[0, \mathbf{s}]}(\mathbf{v}) k(\mathbf{u}, \mathbf{v}) \mathrm{d} \mathbf{u} \mathrm{d} \mathbf{v}
$$

where $k$ is connected to $b$ by $(4.12)$ :

$$
k=b+b^{*}-b \star b^{*},
$$

and $b \in L^{2}\left([0,1]^{2 d}\right)$. The form of covariance follows now from the result in [13], p. 187.

The claims concerning the Radon-Nikodym derivative are obvious given the results of Girsanov representation.

\section{Application to stochastic equations}

We apply the Hitsuda representation to discuss the existence and uniqueness of the solution of a stochastic equation of functional type where the noise term is a (non)fractional Brownian sheet. A rather similar equation (but of a non-functional type) has been studied in [4].

In this section we consider the canonical space $\Omega=C\left([0,1]^{d}\right)$ of continuous sheets (the Brownian and the fractional Brownian sheets admit sample-continuous versions).

Let $\alpha=\left(\alpha(\mathbf{t}, x): \mathbf{t} \in[0,1]^{d}, x \in \Omega\right)$ be a causal or nonanticipative functional. Let $\xi$ be a Gaussian sheet satisfying

$$
\mathrm{d} \xi_{\mathbf{t}}(\omega)=\alpha(\mathbf{t}, \xi(\omega)) \mathrm{d} \mathbf{t}+\mathrm{d} W_{\mathbf{t}}(\omega), \quad \mathbf{P}_{W}-\text { a.s. },
$$

where $W$ is a Brownian sheet. Assume also that the induced Gaussian measure $\mathbf{P}_{\xi}$ satisfies

$$
\mathbf{P}_{\xi}\left(\omega \in \Omega: \int_{[0,1]^{d}} \alpha(\mathbf{t}, \omega)^{2} \mathrm{~d} \mathbf{t}<\infty\right)=1 .
$$


As in the one-parameter case the condition (6.2) implies that $\mathbf{P}_{\xi} \ll \mathbf{P}_{W}$. To see this on just rewrites the proof Theorem 7.3.1 of [8] in multidimensional time and use the multidimensional Girsanov theorem in [3], p. 89. Now, since the measures are Gaussian, we have that $\mathbf{P}_{\xi} \sim \mathbf{P}_{W}$. Hence, by using the Hitsuda representation we have $\xi^{\prime}$, a weak solution of (6.1), given by

$$
\begin{aligned}
\xi_{\mathbf{t}}^{\prime} & =W_{t}-\int_{[0, \mathbf{t}]} \int_{[0, \mathbf{s}]} b(\mathbf{s}, \mathbf{u}) \mathrm{d} W_{\mathbf{u}} \mathrm{d} \mathbf{s} \\
& =W_{t}+\int_{[0, \mathbf{t}]} \int_{[0, \mathbf{s}]} b_{1}(\mathbf{s}, \mathbf{u}) \mathrm{d} \xi_{\mathbf{u}}^{\prime} \mathrm{d} \mathbf{s},
\end{aligned}
$$

where $k_{1}$ is a suitable kernel depending on $\alpha$. As in the one-parameter case, cf [8], p. 234ff, we can now show that if (6.1) admits a strong solution, $\xi^{\prime \prime}$ say, then $\xi^{\prime}=\xi^{\prime \prime}$ $\mathbf{P}_{W}$ (and hence $\mathbf{P}_{\xi}$ ) almost surely. So, we have a complete analogue of Theorem 9.4.2 of $[8]$.

Theorem 6.1 Let $\alpha$ be a nonanticipative functional satisfying (6.2). Then the stochastic equation (6.1) has a Gaussian weak solution if and only if $\alpha$ is of the form

$$
\alpha(\mathbf{t}, \omega)=\int_{[0, \mathbf{t}]} b(\mathbf{t}, \mathbf{s}) \mathrm{d} \omega(\mathbf{u}) \quad \text { Leb } \times \mathbf{P}_{W}-\text { a.e. },
$$

where $b \in L_{V}^{2}\left([0,1]^{2 d}\right)$.

Moreover, if a Gaussian weak solution exists, then so does a Gaussian strong solution, and the latter is unique.

Using Theorem 6.1 and multiparameter version of the technique used in [15] we may prove the following.

Theorem 6.2 Let $Z$ be a fractional Brownian sheet, and let $\alpha$ be a nonanticipating functional satisfying

$$
\int_{[0,1]^{d}} \alpha(\mathbf{t}, \omega) \mathrm{d} \mathbf{t}<\infty \quad \text { for } \quad \mathbf{P}_{Z}-\text { a.a. } \omega .
$$

Then the stochastic equation

$$
\zeta_{\mathbf{t}}=\int_{[0, \mathbf{t}]} \mathcal{K} \mathbf{1}_{[0, \mathbf{t}]}(\mathbf{s}) \alpha(\mathbf{s}, \zeta) \mathrm{d} \mathbf{s}+Z_{\mathbf{t}}
$$

has a Gaussian weak solution if and only if there exists $b \in L_{V}^{2}\left([0,1]^{2 d}\right)$ such that the functional $\alpha$ can be expressed as

$$
\alpha(\mathbf{t}, \omega)=\int_{[0, \mathbf{t}]} b(\mathbf{t}, \mathbf{u}) \mathrm{d} \omega^{*}(\mathbf{u}) \quad \text { Leb } \times \mathbf{P}_{Z}-\text { a.e. },
$$


where we denoted by

$$
\omega^{*}(\mathbf{t})=\int_{[0, \mathbf{t}]} \mathcal{K}^{-1} 1_{[0, \mathbf{t}]}(\mathbf{s}) \mathrm{d} \omega(\mathbf{s}) .
$$

If $H_{i} \leq 1 / 2$ for all $i=1 \ldots d$, then we can write

$$
\alpha(\mathbf{t}, \omega)=\int_{[0, \mathbf{t}]} f(\mathbf{t}, \mathbf{s}) \mathrm{d} \omega(\mathbf{s})
$$

with $f(\mathbf{t}, \cdot)=\mathcal{K}^{-1} b(\mathbf{t}, \cdot)$. Otherwise, if $\max _{i=1 \ldots d} H_{i}>1 / 2$, then there exist functionals that cannot be represented as Wiener integrals with respect to $\omega$.

In any case, if a Gaussian weak solution of (6.4) exists, then a unique Gaussian strong solution exists.

\section{Acknowledgments}

Sottinen was financed by the European Commission research training network DYNSTOCH.

\section{References}

[1] Baudoin, F., and Nualart, D. (2003). Equivalence of Volterra processes. Stochastic Process. Appl. 107, no. 2, 327-350.

[2] Cheridito, P. (2002). Regularizing fractional Brownian motion with a view towards stock price modelling. Ph. D. thesis. ETH Zurich.

[3] Dozzi, M. (1989). Stochastic processes with a multidimensional parameter. Longman Scientific and Technical.

[4] Erraoui, M., Nualart, D., and Ouknine, O. (2002). Hyperbolic stochastic partial differential equations with additive fractional Brownian sheet. Preprint.

[5] Hida, T., and Hitsuda, M. (1993). Gaussian Processes. Translations of Mathematical Monographs, 120, AMS, Providence, RI.

[6] Hitsuda, M. (1968). Representation of Gaussian processes equivalent to Wiener process. Osaka J. Math. 5, 299-312.

[7] Houdre, C., and Villa, J. (2002). An example of infinite dimensional quasi-helix. Contemporary Mathematics.

[8] Kallianpur, G. (1980). Stochastic Filtering Theory. Springer, New York. 
[9] Kallianpur, G., and Oodaira, H. (1973). Non-anticipative representation of equivalent Gaussian processes. Ann. Probability 1, no.1, 104-122.

[10] Pérez-Abreu, V., and Tudor, C. (2002). A transfer principle for multiple stochastic fractional integrals. Bolletin Sociedad Matematica Mexicana, 29 p.

[11] Pipiras, V., and Taqqu, M. (2000). Integration questions related to fractional Brownian motion. Probab. Theory Related Fields 118, no. 2, 251-291.

[12] Pipiras, V., and Taqqu, M. (2001). Are classes of deterministic integrands for fractional Brownian motion on an interval complete? Bernoulli 7, no. 6, 873-897.

[13] Samko, S. G., Kilbas A. A., and Marichev, O. I. (1993). Fractional integrals and derivatives. Theory and applications. Gordon and Breach Science Publishers, Yverdon.

[14] Shepp, L.A. (1966). Radon-Nikodym derivatives of Gaussian measures. Ann. Math. Stat. 37, 321-354.

[15] Sottinen, T. (2004). On Gaussian processes equivalent in law to fractional Brownian motion. J. Theoret. Probab. 17, no. 2, 309-325.

[16] Tudor, C.A. and Viens, F. (2003) Itô formula and local time for the fractional Brownian sheet. Electronic Journal of Probability, 8, paper 14, pp. 1-31.

[17] Tudor C., and Tudor M. (2002). On the multiparameter fractional Brownian motion. Preprint. 